\title{
Nanopoudres de dioxyde de cérium dopé au néodyme : étude d'un capteur de gaz
}

\author{
L. Aneflous ${ }^{1}$, J.A. Musso ${ }^{2}$, J-R. Gavarri ${ }^{2}$, A. Benlhachemi ${ }^{1}$ et H. Benyaich ${ }^{1}$ \\ ${ }^{1}$ Laboratoire de Chimie du Solide, Université Ibn Zohr, Faculté des Sciences, BP. 8, \\ 106 cité Dakhla, 80000 Agadir, Maroc \\ 2 Laboratoire Matériaux \& Microélectronique de Provence (UMR CNRS 6137), Université \\ du Sud-Toulon-Var, BP. 20 132, 83957 La Garde Cedex, France
}

\begin{abstract}
Résumé. Les propriétés structurales et microstructurales, électriques et catalytiques de nouvelles céramiques à base d'oxyde de cérium dopé au néodyme $\mathrm{Ce}_{1-\mathrm{x}} \mathrm{Nd}_{\mathrm{x}} \mathrm{O}_{2-\delta} 0 \leq \mathrm{x} \leq 0,30$ sont étudiées. Ces céramiques sont synthétisées en deux étapes : une méthode sol-gel (à partir de précurseurs oxalates et acétates) qui permet d'obtenir des mélanges homogènes et nanostructurés $(4 \mathrm{~nm})$ suivie d'un frittage à $1600^{\circ} \mathrm{C}$. Les échantillons obtenus sont caractérisés par diffraction des rayons X, analyse thermique différentielle couplée à la thermogravimétrie. Les propriétés électriques des échantillons en fonction de la composition, dans un large domaine de température $\left(40-700^{\circ} \mathrm{C}\right)$, sont étudiées par spectrométrie d'impédance électrique SIE. Des variations non linéaires des résistances et capacités en fonction de la température et de la composition $\mathrm{x}$ sont observées. La conductivité électrique atteint un maximum pour $\mathrm{x}=10 \%$. Les propriétés catalytiques des échantillons ont été étudiées par spectroscopie infra-rouge IRTF. L'étude porte sur la conversion du méthane $\mathrm{CH}_{4}$ en $\mathrm{H}_{2} \mathrm{O}$ et $\mathrm{CO}_{2}$. Les résultats montrent, d'une part, que ces catalyseurs sont très actifs à la température utilisée de $350^{\circ} \mathrm{C}$ et, d'autre part, que leur activité catalytique passe par un maximum pour décroître ensuite.
\end{abstract}

\section{INTRODUCTION}

Les oxydes de terres rares ont été largement utilisés dans la catalyse hétérogène, en raison de leurs propriétés structurales et électroniques, pour améliorer la réactivité, la sélectivité et la stabilité thermique des catalyseurs [1,2]. Le plus intéressant de ces oxydes est certainement l'oxyde de cérium $\mathrm{CeO}_{2}$, appelé « cérine », du fait que ses atomes d'oxygène sont très mobiles et peuvent être facilement éliminés pour conduire à des composés non stoechiométriques, de type $\mathrm{CeO}_{2-\delta}$, $0,070 \leq \delta \leq 0,154$ [3] stables en atmosphère réductrice [4]. Cependant en présence d'oxygène, l'oxyde de cérium retrouve facilement l'état stable $\mathrm{CeO}_{2}$. Cette facilité d'échange de l'oxygène avec les phases gazeuses justifie leur emploi comme capteur de gaz. Les matériaux à base d'oxyde de cérium (films, nanopoudres, micropoudres, composites ...) jouent un rôle capital dans les deux processus les plus intéressants de la catalyse industrielle, à savoir la catalyse à trois voies TWC et la catalyse sur lit fluidisé $[5,6]$.

Ce travail porte sur l'étude des propriétés structurales, microstructurales, électriques et catalytiques de nouvelles céramiques à base d'oxyde de cérium dopé au néodyme $\mathrm{Ce}_{1-\mathrm{x}} \mathrm{Nd}_{\mathrm{x}} \mathrm{O}_{2-\delta} 0 \leq \mathrm{x} \leq 0,30$. 


\section{PARTIE EXPÉRIMENTALE}

Les poudres nanométriques d'oxyde de cérium dopé au néodyme $\mathrm{Ce}_{1-\mathrm{x}} \mathrm{Nd}_{\mathrm{x}} \mathrm{O}_{2-\delta} \quad 0 \leq \mathrm{x} \leq 0,30$ sont synthétisées par une méthode de chimie douce dérivée de la méthode sol-gel [7]. A un mélange, en proportions stœchiométriques, d'oxalate de cérium(+III) et d'acétate de néodyme $(+\mathrm{III})$, on ajoute un solvant organique, l'éthanol absolu. La solution obtenue est agitée pendant 1 heure puis évaporée à $65^{\circ} \mathrm{C}$ jusqu'à obtention d'un xérogel de couleur blanche. Le pH de la solution est compris entre 4 et 5. Le xérogel obtenu subit un traitement thermique à $320^{\circ} \mathrm{C}$ sous air pendant 2 heures. Les poudres nanométriques ainsi obtenues sont broyées puis mises sous forme de pastilles de $13 \mathrm{~mm}$ de diamètre et d'environ $1 \mathrm{~mm}$ d'épaisseur, sous une pression uniaxiale de $5 \mathrm{kbar}$. Les échantillons compactés sont frittés à $1600^{\circ} \mathrm{C}$ pendant 10 heures afin d'obtenir des solutions solides.

\section{RÉSULTATS ET DISCUSSION}

\subsection{Propriétés structurales}

Les résultats de la caractérisation par DRX montrent que ces échantillons cristallisent dans une structure cfc du type fluorite. Aucun pic supplémentaire à la structure de $\mathrm{CeO}_{2}$ n'a été observé. La figure 1 montre le diagramme RX pour la série de poudres nanométriques dopées au néodyme préparées par sol-gel.

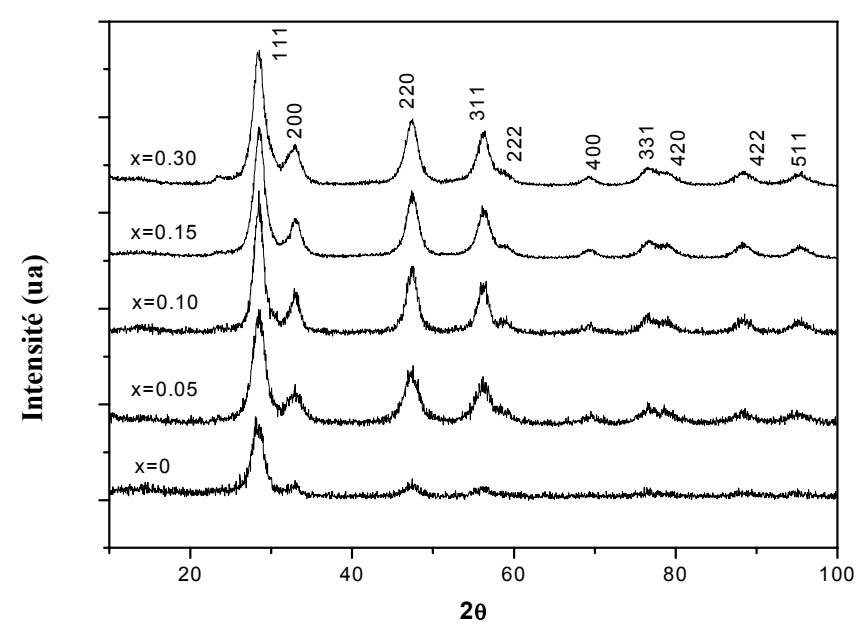

Figure 1. DRX des poudres nanométriques $(1-\mathrm{x}) \mathrm{CeO}_{2} \cdot \mathrm{xNd}_{2} \mathrm{O}_{3}$.

La taille moyenne des cristallites est calculée à partir de la formule de Scherrer : $D_{\text {hkl }}=0,9 \lambda / \beta \cos \theta$ avec $\beta$ l'élargissement du pic déterminé par rapport à un standard et $\mathrm{D}_{\mathrm{hkl}}$ la taille de cristallite en nanomètres. La valeur calculée pour la série d'échantillons (1-x) $\mathrm{CeO}_{2} \cdot \mathrm{xNd}_{2} \mathrm{O}_{3}$ est comprise entre 4,3 $\mathrm{nm}$ et $5,8 \mathrm{~nm}$. On constate qu'elle augmente nettement avec le taux de dopage en $\mathrm{Nd}(+\mathrm{III})$.

Le paramètre de maille est calculé, par la méthode des moindres carrés, avec le logiciel Param. Le paramètre de maille des échantillons frittés à $1600^{\circ} \mathrm{C}$ augmente linéairement en fonction de $\mathrm{x}$ (figure 2) suivant la relation:

$$
\mathrm{a}(\mathrm{x})=541,12+18,63 \mathrm{x}(\mathrm{a} \text { en } \mathrm{pm}) .
$$




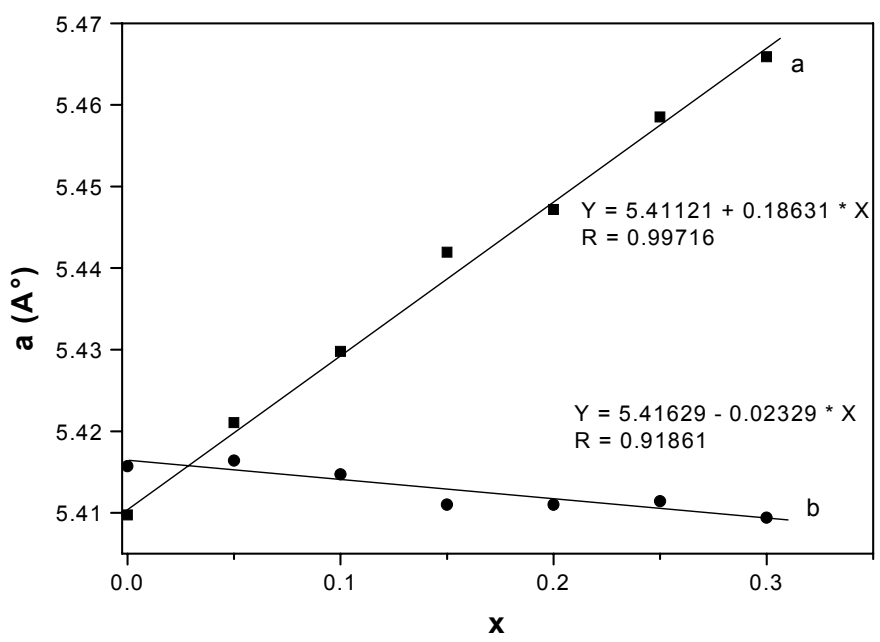

Figure 2. Paramètre de maille $v s$ x pour la cérine dopée au néodyme : a) frittage à $\mathrm{T}=1600^{\circ} \mathrm{C}$; b) sol-gel à $\mathrm{T}=320^{\circ} \mathrm{C}$.

Ce résultat confirme la formation de la solution solide $\mathrm{Ce}_{1-\mathrm{x}} \mathrm{Nd}_{\mathrm{x}} \mathrm{O}_{2-\mathrm{x} / 2} \quad 0 \leq \mathrm{x} \leq 0,30$ en bon accord avec les valeurs des rayons effectifs des cations $\mathrm{Ce}^{4+}\left(\mathrm{r}_{\mathrm{Ce}}{ }^{4+}=111,0 \mathrm{pm}\right.$ en coordinence 8$)$ et $\mathrm{Nd}^{3+}\left(\mathrm{r}_{\mathrm{Nd}}{ }^{3+}=\right.$ $124,9 \mathrm{pm}$ en coordinence 8) [8].

\subsection{Propriétés électriques}

La spectroscopie d'impédance électrique nous a permis d'étudier les propriétés électriques des échantillons en fonction de la composition dans un large domaine de température $\left(40-700^{\circ} \mathrm{C}\right)$. Pour les températures supérieures à $400^{\circ} \mathrm{C}$, les représentations Nyquist ont permis la séparation de trois signaux correspondant aux grains, aux joints de grains et à l'électrode [9].

La conductivité électrique des grains est déterminée en utilisant un circuit équivalent $\mathrm{R} / / \mathrm{C}$. La conductivité électrique atteint un maximum pour $\mathrm{x}=10 \%$ (figure 3 ).

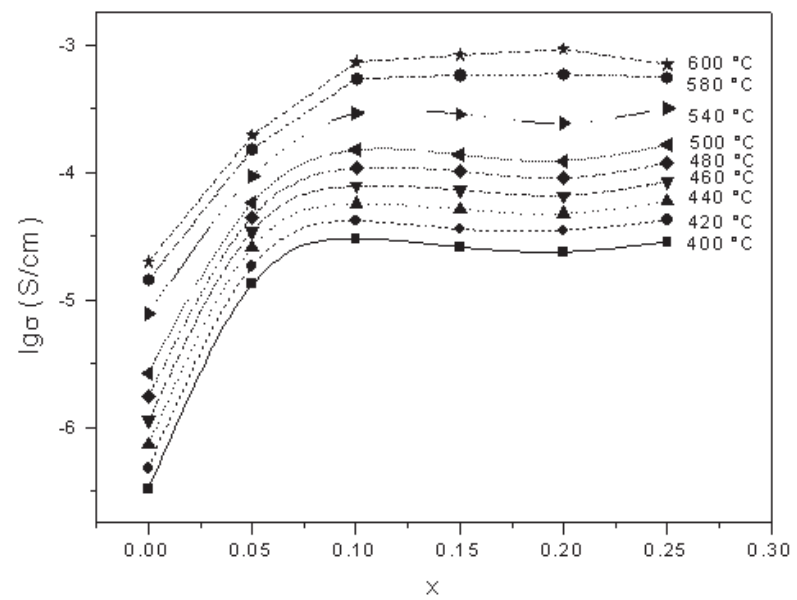

Figure 3. $\lg \sigma=f(x)$ dans le domaine de température $400-700^{\circ} \mathrm{C}$. 
Des variations non linéaires des résistances et capacités en fonction de la température et de la composition x sont également observées. Le modèle CPE (constant phase elements) avec un exposant $n$ non entier a été utilisé pour représenter le circuit équivalent aux joints de grain et aux interfaces avec les électrodes.

\subsection{Propriétés catalytiques}

\subsubsection{Mesures catalytiques}

Le dispositif expérimental utilisé pour réaliser les tests catalytiques est schématisé sur la figure 4. Il comprend trois parties :

a) Alimentation : l'alimentation en gaz est réalisée par l'intermédiaire d'une bouteille contenant 2500 ppm de méthane dilué dans l'air et de deux bouteilles contenant l'une l'oxygène et l'autre l'azote purs. Des débitmètres permettent le réglage des débits. Un mélangeur assure la synthèse du mélange gazeux. Les tests catalytiques sur le méthane sont réalisés avec un débit de $6 \mathrm{~mL} / \mathrm{min}$.

b) Réacteur : on utilise un réacteur tubulaire en inox avec de la laine de verre servant de support au lit catalytique. Ce dernier est fixe et est traversé par les réactifs. Le réacteur est placé dans un four tubulaire permettant de fixer la température de travail $\left(350^{\circ} \mathrm{C}\right)$.

c) Analyse : la cellule d'analyse des gaz sortants est un tube en quartz avec les pastilles de $\mathrm{KBr}$ aux deux extrémités. La cellule est placée dans un spectrophotomètre IRTF. Les spectres sont enregistrés avec une résolution de $8 \mathrm{~cm}^{-1}$; le domaine de longueur d'onde balayé est compris entre $4000 \mathrm{~cm}^{-1}$ et $400 \mathrm{~cm}^{-1}$.

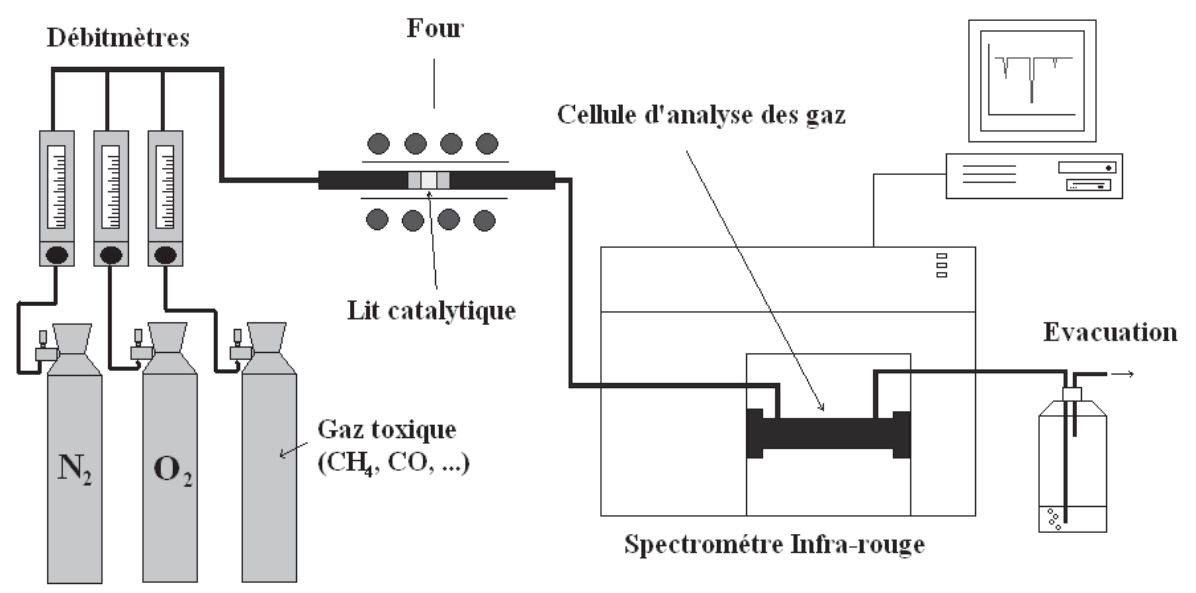

Figure 4. Dispositif expérimental pour la mesure d'activité catalytique.

On effectue une première mesure avec le gaz méthane sans catalyseur, le spectre IR présente les bandes d'absorption à $3020 \mathrm{~cm}^{-1}$ et $1300 \mathrm{~cm}^{-1}$ caractéristiques de la molécule $\mathrm{CH}_{4}$ (figure 5 , spectre a). On effectue une deuxième mesure avec le catalyseur dans le réacteur. Le catalyseur est l'oxyde de cérium pur ou une solution solide $\mathrm{Ce}_{1-\mathrm{x}} \mathrm{Nd}_{\mathrm{x}} \mathrm{O}_{2-\delta}$ sous forme de poudre On chauffe à $350^{\circ} \mathrm{C}$, puis on fait passer le méthane. Le spectre IR des gaz sortants (figure 5, spectre b) présente des bandes d'absorption supplémentaires à $2350 \mathrm{~cm}^{-1}$ et $670 \mathrm{~cm}^{-1}$ caractéristiques de la molécule de $\mathrm{CO}_{2}$ et une large bande d'absorption entre $3730 \mathrm{~cm}^{-1}$ et $3615 \mathrm{~cm}^{-1}$ et une bande à $1580 \mathrm{~cm}^{-1}$ caractéristiques de la molécule d'eau. Ces résultats prouvent la conversion du méthane en dioxyde de carbone et d'eau après passage sur le catalyseur. 


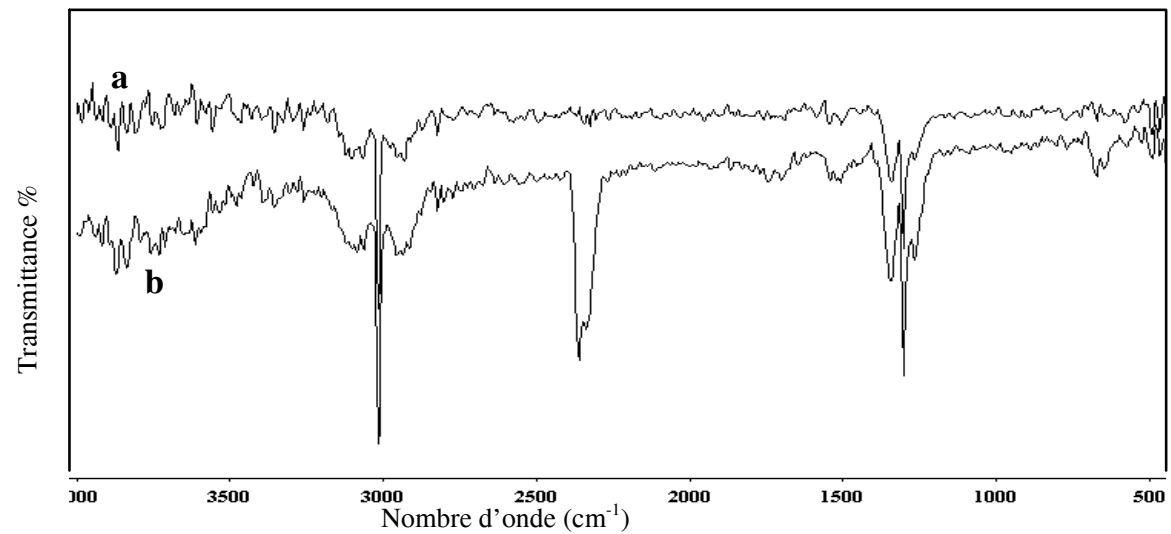

Figure 5. Spectres IR des gaz obtenus avant (a) et après (b) passage du méthane sur le catalyseur $\mathrm{Ce}_{0,95} \mathrm{Nd}_{0,05} \mathrm{O}_{2-\delta}$.

\subsubsection{Réactions}

Le mécanisme de la catalyse hétérogène est complexe et fait intervenir les degrés d'oxydation +III et $+\mathrm{IV}$ du cérium et des atomes d'oxygène mobiles $\mathrm{O}^{*}, \mathrm{O}_{2}^{-}$et $\mathrm{O}_{2}{ }^{2-}$.

Les réactions probables sont :

Oxydation totale ou partielle de $\mathrm{CH}_{4}$ accompagnée de la réduction de $\mathrm{Ce}(+\mathrm{IV})$

$$
\begin{aligned}
& \mathrm{CH}_{4}+\mathrm{Ce}_{1-\mathrm{x}} \mathrm{Nd}_{\mathrm{x}} \mathrm{O}_{2}+(2-\delta / 2) \mathrm{O}_{2} \rightarrow \mathrm{CO}_{2}+2 \mathrm{H}_{2} \mathrm{O}+\mathrm{Ce}_{1-\mathrm{x}} \mathrm{Nd}_{\mathrm{x}} \mathrm{O}_{2-\delta} \\
& \mathrm{CH}_{4}+\mathrm{Ce}_{1-\mathrm{x}} \mathrm{Nd}_{\mathrm{x}} \mathrm{O}_{2}+(1-\delta / 2) \mathrm{O}_{2} \rightarrow \mathrm{CO}+2 \mathrm{H}_{2} \mathrm{O}+\mathrm{Ce}_{1-\mathrm{x}} \mathrm{Nd}_{\mathrm{x}} \mathrm{O}_{2-\delta}
\end{aligned}
$$

Oxydation de $\mathrm{CO}$

$$
\mathrm{CO}+1 / 2 \mathrm{O}_{2} \rightarrow \mathrm{CO}_{2}
$$

Réoxydation du catalyseur

$$
\mathrm{Ce}_{1-\mathrm{x}} \mathrm{Nd}_{\mathrm{x}} \mathrm{O}_{2-\delta}+\delta / 2 \mathrm{O}_{2} \rightarrow \mathrm{Ce}_{1-\mathrm{x}} \mathrm{Nd}_{\mathrm{x}} \mathrm{O}_{2}
$$

Dans nos conditions (température relativement basse $350^{\circ} \mathrm{C}$ ) $\mathrm{CO}$ n'est jamais détecté et l'oxydation de $\mathrm{CH}_{4}$ est totale ; les réactions (2) et (3) sont négligeables devant la réaction (1). En conséquence, la réaction globale considérée est la réaction, aussi bien en phase gazeuse qu'à l'interface gazcatalyseur :

$$
\mathrm{CH}_{4}+2 \mathrm{O}_{2} \rightarrow \mathrm{CO}_{2}+2 \mathrm{H}_{2} \mathrm{O}
$$

\subsubsection{Formulation des résultats}

Le taux de conversion global du réactif $\mathrm{CH}_{4}$ est noté :

$$
\tau\left(\mathrm{CH}_{4}\right)=1-\left[\mathrm{CH}_{4}\right] /\left[\mathrm{CH}_{4}\right]_{0}
$$

$\left[\mathrm{CH}_{4}\right]$ : concentration du réactif à l'instant $\mathrm{t}$,

$\left[\mathrm{CH}_{4}\right]_{0}$ : concentration initiale du réactif. 
Les concentrations sont déduites de l'absorbance du réactif mesurée par IR. Le taux de conversion du méthane calculé pour la solution solide $\mathrm{Ce}_{0,95} \mathrm{Nd}_{0,05} \mathrm{O}_{2-\delta}$ est de $26 \%$.

Les mesures effectuées sur ce catalyseur montrent que la concentration du $\mathrm{CO}_{2}$ produit en fonction du temps (figure 6) atteint un palier au bout de 10 minutes environ. Le catalyseur présente un phénomène de saturation après 26 minutes pour la masse utilisée $(0,30 \mathrm{~g})$. Mis en présence de l'oxygène de l'air, le catalyseur est réactivé. En effet, après réaction, la phase réduite non stœchiométrique $\mathrm{Ce}_{1-\mathrm{x}} \mathrm{Nd}_{\mathrm{x}} \mathrm{O}_{2-\delta}$ est formée; en contact avec l'oxygène de l'air cette phase est oxydée en $\mathrm{Ce}_{1-\mathrm{x}} \mathrm{Nd}_{\mathrm{x}} \mathrm{O}_{2}$ stable en atmosphère oxydante [3].

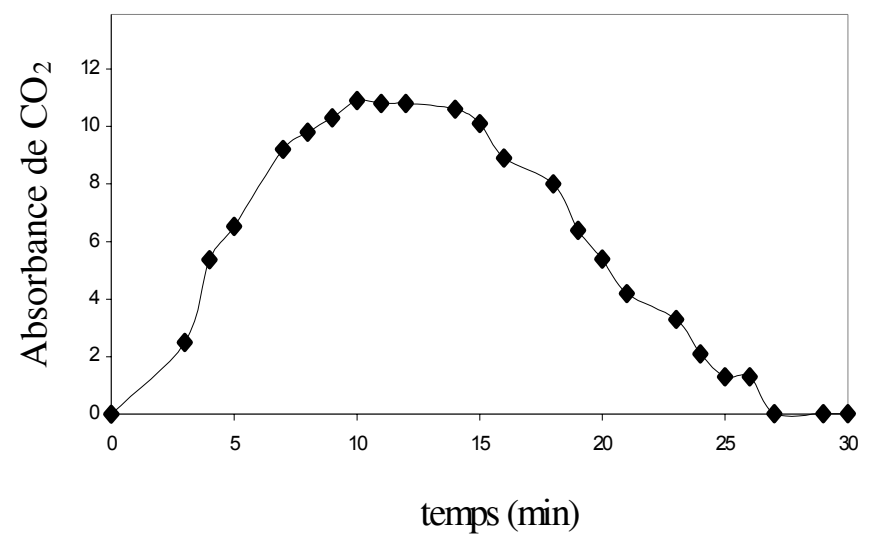

Figure 6. Evolution de l'absorbance de $\mathrm{CO}_{2}$ sur $\mathrm{Ce}_{0,95} \mathrm{Nd}_{0,05} \mathrm{O}_{2-\delta}$.

\section{CONCLUSION}

Les propriétés électriques et catalytiques de nouvelles céramiques à base d'oxyde de cérium dopé au néodyme $\mathrm{Ce}_{1-\mathrm{x}} \mathrm{Nd}_{\mathrm{x}} \mathrm{O}_{2-\delta} 0 \leq \mathrm{x} \leq 0,30$ ont été étudiées Les évolutions observées de la conductivité électrique avec la température et la composition confirment les résultats de la littérature, en terme d'accroissement des défauts isolés et de concentration de défauts liés à la percolation des porteurs de charges, au-delà d'une certaine fraction de néodyme $(10 \%)$. Les tests catalytiques par spectrométrie IR montrent que ces matériaux transforment le gaz toxique $\mathrm{CH}_{4}$ en $\mathrm{CO}_{2}$ et $\mathrm{H}_{2} \mathrm{O}$; le taux de conversion est relativement important aux basses températures $\left(25 \%\right.$ à $\left.350^{\circ} \mathrm{C}\right)$. Des études seront menées avec d'autres gaz toxiques $\left(\mathrm{NH}_{3}, \mathrm{SO}_{\mathrm{x}, \mathrm{x}}=2\right.$ ou $\left.3, \mathrm{CO}\right)$.

\section{Références}

[1] Yingli B., Kaiji Z., Yutao J.,Chiwen T., Xiangguong Y., Catalysis, 39 (1988) 185-190.

[2] Maitra A.M., Campbell I., Tyler R.J., Applied Catalysis A: General, 85 (1992) 27-46.

[3] Riess I., Ricken M., Nolting J., J. Solid State Chem., 57 (1985) 314-322.

[4] Korner R., Riken M., Nolting J., I. Riess, J. Solid State Chem., 16 (1989) 136.

[5] Trovarelli A., Catal. Rev.-Sci. Eng. 38 (1996) 439.

[6] Kapar J., Fornasiero P., Graziani M., Catalysis Today, 50 (1999) 285-298.

[7] Villain S., Leroux Ch., Musso J.A., Gavarri J-R., J. Metast. Nano. Mater., 12 (2002) 59.

[8] Shannon R.D., Prewitt C.T., Acta Crystallogr. 32A (1976) 751.

[9] Aneflous L., Musso J.A., Villain S., Gavarri J-R.., Benyaich H., J. Solid State Chem., 177 (2004) $856-865$. 